\title{
Effects of Solvents on the Electronic and Molecular Properties of 4- ((2-Methyl-4-Nitrophenyl) Imino Methyl)Phenol
}

\author{
A.A Akande ${ }^{1}$, N.O Obi-Egbedi ${ }^{2}$ and N.D Ojo ${ }^{3}$ \\ ${ }^{1-3}$ Department of Chemistry \\ University of Ibadan \\ Ibadan, Oyo State \\ Nigeria.
}

\begin{abstract}
Schiff bases are known to possess antiviral, antifungal, antibacterial, enzymatic and other significant biological properties. They also chelate metals to form complexes that have been used in industrial processes. Schiff bases are also important in modern energy applications due to their photometric and thermochemical properties. The basis of their applications depends on the molecular and electronic properties hence it is important to study how solvents perturb these properties. Therefore, this work is aimed at studying the electronic and molecular properties of a Schiff base. The effects of solvents on 4-(((2-methyl-4nitrophenyl)imino)methyl)phenol was investigated both experimentally and theoretically. Experimentally, the UV-Visible spectrophotometer was used to obtain the absorption spectra of the compound in tetrahydrofuran, dichloromethane, propan-2-,ol, propan-1-ol, ethanol and methanol. Time-Dependent Density Functional Theory (TDDFT) and B3LYP with the 6-311++G(d,p) basis set was used to model and calculate the dipole moment, polarizability and excitation energies in vacuum, cyclohexane, methanol and ethanol using a computational software. In the experimental studies, four bands were observed and designated I, II, III and IV in methanol and ethanol. However three bands were observed in propan-1-ol and propan-2-ol, and two bands in dichloromethane and tetrahydrofuran. A red shift was observed in Bands I and II while a blue shift was observed for Band III as solvent polarity increases. The intensity of the bands increased with increase in solvent polarity. The dipole moment and polarizability calculated using TDDFT increased with increasing solvent polarity. This may indicate ease of charge separation and distortion of the electron cloud of the molecule as polarity of solvent increases. On the basis of the observations of the transition energy and wavelength with solvent polarity, the compound can be classified to have both $n \rightarrow \pi^{*}$ and $\pi \rightarrow \pi^{*}$ transitions.
\end{abstract}

Key words: Solvents, Oscillator strength, Dipole moment, Time-Dependent Density Functional Theory, Polarity.

\section{INTRODUCTION}

Schiff bases are formed from the condensation reaction between an aldehyde (or ketone) and a primary amine. The aldehydes and ketones contain a carbonyl group, $\mathrm{C}=\mathrm{O}$ and this group is replaced with the $\mathrm{C}=\mathrm{N}-\mathrm{R}$ present in the $\mathrm{Schiff}$ bases. This reaction may occur either through an acid or base catalyzed pathways. Although if an acid pathway is followed, it must be at mild $\mathrm{pH}$ to avoid hydrolysis of the product. These compounds are often called Schiff bases when they are used as ligands to form coordination complexes with metal ions. These complexes may occur naturally or may be synthesised artificially. When used as ligands, they have strong coordinative ability and are good chelating agents. Since, they mostly are polydentate ligands, almost all of the Schiff bases can form 1:1 complexes with transition metals and these complexes are very stable. Due to this, a number of Schiff base ligands have been used as cation carriers in potentiometric sensors, good ion carriers for anion selective sensors as effective corrosion inhibitors, in enzyme preparation and as catalysts in certain biological systems [1]. Schiff bases are becoming increasingly important in the medical and pharmaceutical fields. This is because they show variety of biological activities. They are known for their anti-inflammatory, anticancer, antioxidant, anticonvulsant, analgesics, among many others. The imine functional group plays a unique role in the biological activities of the compound. It is suspected that the nitrogen atom of the azomethine is involved in the formation of a hydrogen bond with the active centres of a cell constituents and this allows the compound to interfere with the normal cell processes thereby bringing about the biological activities [2]. Schiff bases and its metal complexes synthesised have been used as mordants in dyeing techniques. Abuamer et al synthesised dyes using salicylaldehyde and 2-aminobenzyl alcohol or o-aminophenol in hot ethanolic solution as starting materials. A yellow and orange solid Schiff 
bases were formed and these dyes had extremely good fastness properties [3]. Copper complexes of Schiff bases were also explored and the dyes produced have excellent light resistance, storage ability, ability to give fast colours and even ability to not degrade in acidic gases such as carbondioxide [4]. Schiff bases also possess photometric and thermochemical properties making them useful in modern energy applications such as production of solar filters, microelectronic devices, photodetectors, photostabilizers, and optical switches [5].

Schiff bases are becoming increasingly important in the scientific world. Particular attention have been given to them because they have found a variety of application in various fields ranging from the medical field to the engineering field and even in living organisms. One of the interesting applications of Schiff bases are their biological activities. Studies have shown that Schiff bases possess antibacterial, antifungal, enzymatic, antiviral, anticancer and antimalarial properties. They have also found applications as non-linear optics materials, catalysts, solar cells and in photodynamic therapy. These applications justify why they are studied. Schiff bases have good emission and optical properties as well. Understanding the electronic and molecular properties of a Schiff base will give an idea on where it can be applied.

The aim of this project was to investigate the effect of solvent perturbation on the absorption properties of the synthesised Schiff base, 4-(((2-methyl-4-nitrophenyl)imino)methyl)phenol. This was carried out using the UV-Visible spectroscopy technique. The objectives of this research were to study the effect of solvent perturbation on absorption bands of the Schiff base and to correlate results obtained from experimental method to those obtained from computational results.

\section{MATERIALS AND METHOD}

The compound studied in this work is a Schiff base, 4-(((2-methyl-4-nitrophenyl)imino)methyl)phenol synthesised using 4-hydroxylbenzaldehyde and 2-methyl-4-nitroaniline as starting materials. The compound is a light yellow solid with molecular formula $\mathrm{C}_{14} \mathrm{H}_{12} \mathrm{~N}_{2} \mathrm{O}_{3}$ and molecular weight $256.26 \mathrm{gmol}^{-1}$. It had been synthesised and characterised by researchers in A5 Lab, Chemistry department, University of Ibadan.

Six solvents of varying polarity were used, namely methanol, ethanol, propan-1-ol, propan-2-ol, tetrahydrofuran and dichloromethane. Ethanol, methanol and dichloromethane used had been purified by distillation and their distillation process had been done in the laboratory following the standard guidelines. All weighing procedures were carried out using OhausPioneer ${ }^{\mathrm{TM}}$ Analytical weighing balance and the method of difference was used to determine the weight of sample.

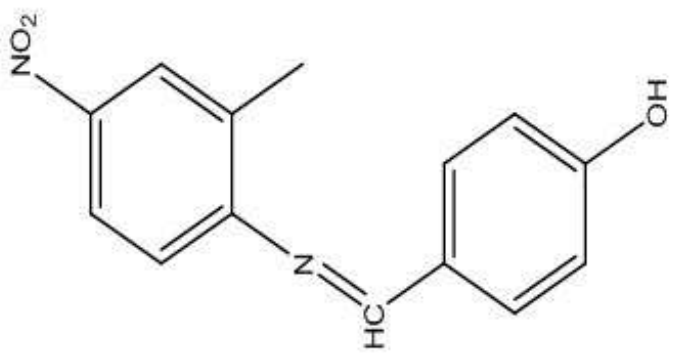

Figure 1: 4-(((2-methyl-4-nitrophenyl)Imino)methyl)Phenol

\subsection{Experimental Procedure}

One to two milligrams of the Schiff base under study was weighed into a clean, dry $10 \mathrm{ml}$ standard flask and dissolved in appropriate solvent. After dissolution, the solvent was used to make it up to mark and the concentration of the stock solution was calculated for each of the six solvents. Aliquots of the stock solution of each solvent were taken and diluted appropriately to be used for spectrometric studies. The concentration of each working solution obtained from the aliquots was also calculated. This procedure was carried out for each of the solvent listed above.

The absorption spectra were recorded using Perkin Elmer ${ }^{\circledR}$ precisely, Lambda 25 UV/Vis Spectrometer. The instrument is a double-beam spectrophotometer having the sample and reference compartments each having a cuvette with a pathlength of one milliliter. The electronic absorption study was carried out within the concentration range of $10^{-4}$ to $10^{-6}$ in order to ensure that the maximum absorbance does not exceed one. Absorbance of the sample was recorded over a wavelength range of $190 \mathrm{~nm}$ to 700 $\mathrm{nm}$, however the solutions read were absorbed between $190 \mathrm{~nm}$ and $460 \mathrm{~nm}$. The graph of absorbance against wavelength was plotted over a wavelength range of $190 \mathrm{~nm}$ to $460 \mathrm{~nm}$. The molar extinction coeffiecient, $\varepsilon$ and the wave number, $\bar{v}$, were calculated. The graph of molar extinction against wavenumber was plotted and and stacked for comparison. 
The oscillator strength of each band was also calculated as a measure of the intensity of absorption of molecules. This calculation was done from the maximum molar extinction coefficient and wavelength using the equations:

$$
f=\frac{4.321 \times 10^{-9}}{n} \int \varepsilon_{(\bar{v})} d \bar{v}---\cdot--\cdot-----(1)
$$

where, $f$ is the oscillator strength, $\varepsilon_{\max }$ is maximum absorption coefficient, and $\int \varepsilon_{(\bar{v})} d \bar{v}$ describes the area of the Guassian distribution curve with the assumption of symmetrical bands.

\subsection{Computational Details}

Quantum chemical calculations were carried out in Gaussian '09 programme package using Time Dependent Density Functional Theory (TDDFT). The molecule was built in vacuum first and the geometry optimized using Becke-3-Lee-Yang-Parr (B3LYP) method with 6-311++G(d,p) basis set. The Time Dependent Density Functional Theory (TDDFT) is an extension of the Density Functional Theory (DFT). It shows that the time-dependent wave function is equivalent to the time-dependent electronic density and the density is a simple function that depends solely on three variables $\mathrm{x}, \mathrm{y}$ and $\mathrm{z}$. TDDFT (as the DFT) is also an $a b$ initio method and ab initio methods are more accurate than semiempiricalmethods due to their pure mathematical approach. These reasons made TDDFT a more desirable quantum mechanical theory to use in the calculation of energies of excited states of the compound.

The method was first carried out in the ground state using B3LYP and also using 6-311++g(d,p) as the basis set. 6$311++G(d, p)$ describes diffuse functions on heavy atoms and hydrogen and doubly polarized split valence basis set. Although it takes a longer run time, it produces better results than single polarized split valence basis set and so it was preferred in our calculations. Becke-3-Lee-Yang-Parr (B3LYP) is a type of hybrid exchange-correlation functional and was preferred to other methods like Local Density Approximation (LDA) because they provide better results for reaction chemistry calculations. Transitions of various excited states were also calculated. The polarizabilities of the compound in vacuum and the different solvents were calculated from their coordinates $\mathrm{xx}$, yy and $\mathrm{zz}$ of the quadrupole moment using the Equation (3.4):

$$
\text { Polarizability, } \alpha=\frac{1}{3}\left(\alpha_{x x}+\alpha_{y y}+\alpha_{z z}\right)
$$

Where, $\alpha_{\mathrm{xx}}=$ quadrupole moment in the XX direction, $\alpha_{\mathrm{yy}}=$ quadrupole moment in the $\mathrm{YY}$ direction, $\alpha_{\mathrm{zz}}=$ quadrupole moment in the $\mathrm{ZZ}$ direction and $\alpha=$ Polarizability.

Values of parameters such as oscillator strength, transition moments, transition energy in electron volts (eV) and wavelength in nanometers which had been computed theoretically were also recorded.

\section{RESULTS AND DISCUSSION}

\subsection{Solvent Effect on Transition Energy And Wavelength}

Four bands were observed in methanol and ethanol as reported in Table 1. These bands were designated Band I $\left(\mathrm{S}_{0} \rightarrow \mathrm{S}_{1}\right)$, Band II $\left(S_{0} \rightarrow S_{2}\right)$, Band III $\left(S_{0} \rightarrow S_{3}\right)$ and Band IV $\left(S_{0} \rightarrow S_{4}\right)$ in order of increasing energy. Band I was the most intense in all of the solvents. This was followed by Band III in all solvents except ethanol and methanol where a fourth band was observed. Band II appeared as very broad, structureless and of the lowest intensity. This may be attributed to borrowed intensity from Band I.

Band I and Band II were observed in all six solvents. However, Band III and Band IV were not observed in tetrahydrofuran and dichloromethane. Band IV was also absent in propan-2-ol and propan-1-ol. These observations may be attributed to the non-transparency of the solvent to UV light in that region. Tetrahydrofuran and dichloromethane have the highest cutoff wavelengths and so they absorb UV light at the positions where Band III and Band IV should be observed, hence no band was observed at all at these positions. Propan-1-ol and propan-2-ol have higher cutoff wavelengths than ethanol and methanol but lower than those of dichloromethane and tetrahydrofuran hence Band III was observed in propan-1-ol and propan-2-ol but Band IV was not.

Band positions depend on the solute and the solvent in which it is dissolved. Table 1 shows that Band I of 4-(((2-methyl4-nitrophenyl)imino)methyl)phenol in all of the six solvents has high molar absorptivity and oscillator strengths. The implication of this is that the bands are intense as oscillator strength is a measure of the band's intensity. This is probably due to the extension of conjugation in the Schiff base through the delocalization of $\pi$-electrons or due to intramolecular charge transfer involving charge redistribution in the excited molecule which produces a very large excited state dipole moment. The hydroxyl group may increase the electron density of the ring by pushing electrons towards the ring through mesomeric effect and the nitro group being an electron withdrawing group pulls electron cloud away from the ring causing the redistribution of charge. The presence of the 
hydroxyl group on one of the benzene rings as an auxochrome could also lead to the extension of conjugation. These could account for the bathochromic shift in wavelength as solvents polarity increases.

A general trend was observed with the highly intense Band I.As solvent polarity increases, a decrease in wavenumber was observed. This implies that there is a red shift of the $S_{0} \rightarrow S_{1}$ transition with increasing solvent polarity. Therefore, it can be said that Band I is essentially from a $\pi \rightarrow \pi^{*}$ transition. The same trend was also generally observed in Band II although there is a slight deviation with dichloromethane and tetrahydrofuran in these bands. A decrease in wave number was also observed as solvent polarity increases in the fourth transition energy band. Hence, it can be said that increase in polarity of solvent in this experiment caused a lower energy to be used since the $\pi \rightarrow \pi *$ transition allows polar solvents to stabilize the excited state relative to the ground state due to electrostatic interaction. Energy of absorption is inversely proportional to wavelength, therefore the bands of absorption moves to a longer wavelength as energy is reduced. This shift to a longer wavelength is known as bathochromic shift or a red shift.The less polar solvents do not stabilize the excited state as much as the polar solvents and so they absorb using more energy and cause movement of bands to a shorter wavelength.

Band III deviates from the trend. A decrease in wavelength is observed as polarity of solvents increases from the less polar propan-2-ol to the highly polar methanol. Hence, a red shift is observed for this band. This indicates an $\mathrm{n} \rightarrow \pi^{*}$ transition which is possible due to the lone pairs of electrons on the oxygen of the hydroxyl group which may undergo protonation with methanol and other highly polar solvents through hydrogen bonding. 
International Journal of Advances in Scientific Research and Engineering (ijasre), Vol 5 (8), August-2019

Table 1: This table details of the several bands observed with each solvent, the wavenumber $(\bar{v})$ in $\mathrm{cm}^{-1}$ with corresponding molar absorptivity $(\varepsilon)$ in $\mathrm{M}^{-}$ ${ }^{1} \mathrm{~cm}^{-1}$ and the oscillator strength (f) of 4-(((2-methyl-4-nitrophenyl)imino)methyl)phenol in the six different solvents; Methanol, Ethanol, propan-2-ol, propan-1-ol, Dichloromethane and Tetrahydrofuran.

\begin{tabular}{|c|c|c|c|c|c|c|c|c|c|c|c|c|}
\hline \multirow[t]{2}{*}{ SOLVENT } & \multicolumn{3}{|c|}{ BAND I } & \multicolumn{3}{|c|}{ BAND II } & \multicolumn{3}{|c|}{ BAND III } & \multicolumn{3}{|c|}{ BAND IV } \\
\hline & $\begin{array}{c}\bar{v} \\
\left(\mathrm{~cm}^{-1}\right)\end{array}$ & $\begin{array}{c}\varepsilon \\
\left(\mathrm{M}^{-1} \mathrm{~cm}^{-1}\right)\end{array}$ & $\mathrm{F}$ & $\begin{array}{c}\bar{v} \\
\left(\mathrm{~cm}^{-1}\right)\end{array}$ & $\begin{array}{c}\varepsilon \\
\left(\mathrm{M}^{-1} \mathrm{~cm}^{-1}\right)\end{array}$ & $\mathrm{F}$ & $\begin{array}{c}\bar{v} \\
\left(\mathrm{~cm}^{-1}\right)\end{array}$ & $\begin{array}{c}\varepsilon \\
\left(\mathrm{M}^{-1} \mathrm{~cm}^{-1}\right)\end{array}$ & $\mathrm{f}$ & $\begin{array}{c}\bar{v} \\
\left(\mathrm{~cm}^{-1}\right)\end{array}$ & $\begin{array}{c}\varepsilon \\
\varepsilon \\
\left(\mathrm{M}^{-1} \mathrm{~cm}^{-1}\right)\end{array}$ & f \\
\hline Methanol & 26666.7 & 16160.5 & 0.65 & 36297.6 & 9861.9 & 0.21 & 46083.0 & 12063.6 & 0.15 & 50505.1 & 15014.3 & 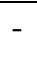 \\
\hline Ethanol & 26954.2 & 16682.5 & 0.69 & 36363.6 & 12631.1 & 0.38 & 46296.3 & 15734.5 & 0.52 & 50761.4 & 22391.7 & - \\
\hline Propan-2-ol & 27100.3 & 20352.7 & 0.97 & 36363.6 & 12631.1 & 0.40 & 45558.1 & 17449.1 & 0.54 & - & - & - \\
\hline Propan-1-ol & 27248.0 & 21257.9 & 0.84 & 37105.8 & 11168.4 & 0.44 & 45871.6 & 18574.0 & 0.69 & - & - & 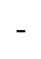 \\
\hline Dichloromethane & 28612.3 & 19959.2 & 0.68 & 38759.7 & 14791.9 & 0.37 & - & - & - & - & - & 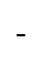 \\
\hline Tetrahydrofuran & 27662.5 & 17923.0 & 0.50 & 37664.8 & 14299.3 & 0.47 & - & - & - & - & - & - \\
\hline
\end{tabular}




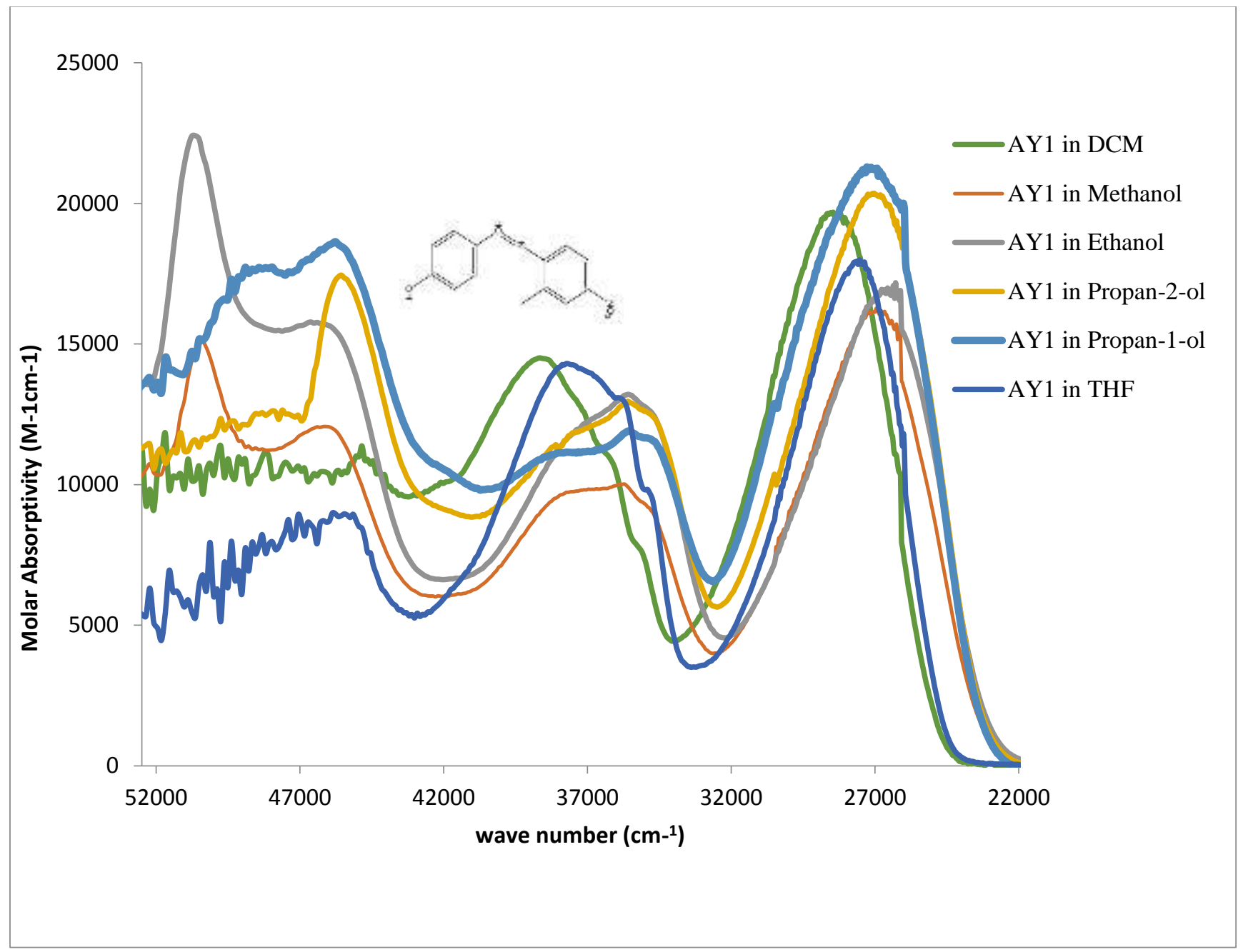

Figure 2: Absorption spectra of 4-(((2-methyl-4-nitrophenyl)imino)methyl)phenol showing the comparison between solute bands in different media expressed as graph of molar absorptivity against wavenumber. The colour of each solvent is designated by the legends.

\subsection{Calculated Electronic Properties of the Compound}

When the first ten excited states of 4-(((2-methyl-4-nitrophenyl)imino)methyl)phenol in vacuum, cyclohexane, methanol and ethanol were compared, it was observed that the lowest energy transition band progressively shifts to longer wavelengths as solvent polarity increases. Hence, Band I has its longest wavelength in ethanol and methanol which are of similar polarity, a shorter one is cyclohexane which is less polar and its shortest wavelength in vacuum which is the nonpolar medium. This infers that there is a red shift as polarity of solvent increases. It was observed that the HOMO $\rightarrow$ LUMO have the lowest transition energies in all four solvents and the energy required to move from HOMO to LUMO progressively decreases as polarity of solvents increases. Again, this is an indication that the excited state is more stabilized than the ground state with increasing polarity; therefore the transition energy needed is reduced. This results to a bathochromic shift as was observed in the experimental Band I. Generally, the computational studies have shown that a bathochromic shift occurs as polarity of solvent increases as seen in the experimental results. This may also be due to the stabilization of the excited state in a $\pi \rightarrow \pi^{*}$ transition by the polar solvents causing a decrease in the energy gap and consequently, an increase in wavelength. This theory corresponds with that obtained from the experimental studies.

Parameters such as the dipole moment and polarizabilities of the compound in the different environments were determined from the computational studies. This is shown in Table 2 and it was observed that dipole moments 
and polarizabilities of the compound increases with increasing solvent polarity. The polarizability is a measure of how easily the electron cloud of the molecule is distorted by the electric field of its environment while the dipole moment is a product of the distance between the charges and the spatial distribution of the charges in a molecule. The high dipole moment could be as a result of good charge separation.

Investigation of the electronic absorption properties of 4-(((2-methyl-4-nitrophenyl)imino)methyl)phenol in cyclohexane could not be carried out experimentally because the Schiff base exhibited solubility problems in the solvent due to its low polarity. However, calculations of the compound in cyclohexane were carried out theoretically using computational techniques.

Table 2: Dipole moments in (D) and Polarizabilities in (a.u) of 4-(((2-methyl-4-nitrophenyl)imino)methyl)phenol in cyclohexane, ethanol, methanol and in vacuum.

\begin{tabular}{ccc}
\hline Medium & Dipole moment $(\mathbf{D})$ & Polarizability (a.u) \\
\hline Vacuum & 8.15 & 117.85 \\
Cyclohexane & 9.20 & 118.72 \\
Ethanol & 10.64 & 120.14 \\
Methanol & 10.68 & 120.19
\end{tabular}

\section{CONCLUSION}

The effect of solvent on the electronic absorption spectra of a Schiff base, 4-(((2-methyl-4nitrophenyl)imino)methyl)phenol have been investigated in this report and the studies have shown that solvents have prominent effect on the transition energy and intensity of the absorption bands. The property of the solvents that affect the absorption spectra most is the polarity of the solvent. The polar solvents stabilized the excited state relative to the ground state due to electrostatic interaction and so the transition energies of the first two bands were reduced causing a bathochromic shift as solvent polarity increases. This could be attributed to a $\pi \rightarrow \pi^{*}$ transition. Band III however showed a blue shift which could be attributed to an $n \rightarrow \pi^{*}$ and is probably due to the presence of the hydroxyl oxygen that can undergo protonation with polar solvents. This causes the ground state to be more stabilized relative to the excited state.

Therefore, solvent perturbation of the compound may allow the bands to be classified as $\pi \rightarrow \pi^{*}$ or $\mathrm{n} \rightarrow \pi^{*}$ transitions.

\section{REFERENCES}

1. K. Brodowska and E. Łodyga-chruścińska 2014. Schiff bases - interesting range of applications in various fields of science. CHEMIK International68.2: 129-134.

2. H. Abdul, R. Mariya, U. Maliha, A. Syed and M. Khalid. 2017. Schiff bases in medicinal chemistry: a patent review (2010-2015). Expert Opinion on Therapeutic Patents 27:1, 63-79.

3. K. M. Abuamer, A.A. Maihub, M. M. El-Ajaily, A. M. Etorki, M.M. Abou-Krisha, and M. A. Almagani. 2014. The Role of Aromatic Schiff Bases in the Dyes Techniques. International Journal of Organic Chemistry 4: 7-15.

4. S. Kumar, D.N. Dhar and P. N. Saxena. 2009. Applications of metal complexes of Schiff bases- A review. Journal of scientific and industrial research 86:181-182.

5. A. Rauf, A. Shah, K. S. Munawar, A. A. Khan, R. Abbasi, M.A. Yameen, A. M. Khan, A. R. Khan, I. Z. Qureshi and H. B. Kraatz. 2017. Synthesis, spectroscopic characterization, DFT optimization and biological activities of Schiff bases and their metal (II) complexes. Journal of Molecular Structure doi: 10.1016/j.molstruc.2017.05.098. 